\title{
Article \\ $\gamma$-Aminobutyric Acid Suppresses Iron Transportation from Roots to Shoots in Rice Seedlings by Inducing Aerenchyma Formation
}

\author{
Changhua Zhu ${ }^{\dagger}$, Qi Qi ${ }^{\dagger}$, Huijiao Niu, Jiaqi Wu, Na Yang and Lijun Gan *[C \\ College of Life Sciences, Nanjing Agricultural University, Nanjing 210095, China; zch@njau.edu.cn (C.Z.); \\ 2018816118@njau.edu.cn (Q.Q.); 13135403358@126.com (H.N.); 23217212@njau.edu.cn (J.W.); \\ yangna@njau.edu.cn (N.Y.) \\ * Correspondence: ganlj@njau.edu.cn \\ t These authors contributed equally to this work.
}

Citation: Zhu, C.; Qi, Q.; Niu, H.;

Wu, J.; Yang, N.; Gan, L.

$\gamma$-Aminobutyric Acid Suppresses Iron Transportation from Roots to Shoots in Rice Seedlings by Inducing Aerenchyma Formation. Int. J. Mol. Sci. 2021, 22, 220. https://doi.org/ $10.3390 /$ ijms 22010220

Received: 18 November 2020 Accepted: 24 December 2020 Published: 28 December 2020

Publisher's Note: MDPI stays neutral with regard to jurisdictional clai$\mathrm{ms}$ in published maps and institutional affiliations.

Copyright: (C) 2020 by the authors. Licensee MDPI, Basel, Switzerland. This article is an open access article distributed under the terms and conditions of the Creative Commons Attribution (CC BY) license (https:// creativecommons.org/licenses/by/ $4.0 /)$.

\begin{abstract}
Aminobutyric acid (GABA) is a widely distributed non-protein amino acid mediated the regulation of nitrate uptake and $\mathrm{Al}^{3+}$ tolerance in plants. However, there are few reports about the involvement of GABA in the regulation of iron (Fe) acquisition and translocation. Here, we show that GABA regulates Fe homeostasis in rice seedlings. Exogenous GABA decreased the chlorophyll concentration in leaves, with or without Fe supply. Over-expression of glutamate decarboxylase $(G A D)$ gene, coding a crucial enzyme of GABA production, elevated endogenous GABA content and caused more leaf chlorosis than wild type (Nipponbare). GABA inhibited Fe transportation from roots to shoots and GABA application elevated the expression levels of Fe deficiency (FD)-related genes under conditions of Fe-sufficiency (FS), suggesting that GABA is a regulator of Fe translocation. Using Perls' blue staining, we found that more ferric iron $\left(\mathrm{Fe}^{3+}\right)$ was deposited in the epidermal cells of roots treated with GABA compared with control roots. Anatomic section analysis showed that GABA treatment induced more aerenchyma formation compared with the control. Aerenchyma facilitated the oxidization of soluble ferrous iron $\left(\mathrm{Fe}^{2+}\right)$ into insoluble $\mathrm{Fe}^{3+}$, resulted in Fe precipitation in the epidermis, and inhibited the transportation of Fe from roots to shoots.
\end{abstract}

Keywords: $\gamma$-aminobutyric acid; iron translocation; aerenchyma formation; iron-deficiency-related genes

\section{Introduction}

Iron $(\mathrm{Fe})$ is an important micro-element for growth and development in plants, as it is required in many processes, including photosynthesis and respiration [1,2]. Although the earth is rich in $\mathrm{Fe}$, it is scarcely available to plants, especially in alkaline soils, due to the formation of insoluble ferric iron $\left(\mathrm{Fe}^{3+}\right)$ precipitates, which results in Fe deficiency (FD) $[1,2]$. FD is one of the largest problems in crop cultivation, as it limits plant productivity. On the other hand, higher concentrations of ferrous iron $\left(\mathrm{Fe}^{2+}\right)$ present a risk, especially when plants are cultivated in acidic conditions [3,4]. Thus, an understanding of the mechanisms of Fe acquisition and translocation in plants is critical for the breeding of crops with better growth under different Fe conditions.

Plants have two ways for Fe uptake, referred to as strategies I and II. Non-graminaceous monocots and dicots use strategy I, and graminaceous monocots use strategy II, to deal with FD in soil [1,2]. Ferric reductase, iron-regulated transporters (IRTs), and acidification of the rhizosphere are involved in root Fe uptake employing strategy I. In contrast, graminaceous plants produce the natural $\mathrm{Fe}^{3+}$ chelators mugineic acid (MAs) family phytosiderophores to solubilize $\mathrm{Fe}^{3+}[1,2]$. In rice, MAs are secreted from roots to the rhizosphere via an exporter TOM1 [5], and the $\mathrm{Fe}^{3+}-\mathrm{MA}$ complex is then absorbed into the roots by yellow stripe/yellow stripe 1-like (YSL) transporters, such as OsYSL15 [6,7]. Research has shown that FD induces MAs accumulation, and that nicotianamine synthase (NAS), nicotianamine 
aminotransferase (NAAT), and deoxymugineic acid synthase (DMAS) are responsible for MAs biosynthesis from S-adenosylmethionine (SAM) [1]. All MAs share this synthetic pathway to deoxymugineic acid (DMA), which is then used to produce other MAs [8].

Previous studies have shown that citrate, nicotianamine (NA), and DMA are vital regulators in Fe transport through xylem [9]. In addition, YSL can also regulate the Fe transportation in the plant body, for example, OsYSL2 is a transporter of Fe(II)-NA and is involved in transportation of Fe in phloem [10]. Rice plants in submerged paddy fields also possess an $\mathrm{Fe}^{2+}$-transporter system, and several genes have been identified associated with $\mathrm{Fe}^{2+}$ transportation, such as OsIRT1, OsIRT2, and OsNRAMP1 [11-13].

$\gamma$-Aminobutyric acid (GABA) is a widely distributed non-protein amino acid found in nature [14]. GABA concentrations have been found to increase as part of plant responses to adverse abiotic stresses, such as drought, anoxia, heat stress, salt, and low temperature $[15,16]$. Glutamate decarboxylase (GAD) is responsible for the conversion of glutamate to GABA [17]. GABA shunt involving the metabolism of GABA to succinic semialdehyde and then succinate and fed into the tricarboxylic acid cycle also affect GABA level in plant [18]. The half-life of $\mathrm{D}_{6}$-GABA was reported to be $1.3 \mathrm{~h}$ and it can be quickly converted to succinate [19].

GABA is also responsible for regulating the responses of plants to toxicity of metal elements, such as the possible regulation of arsenite, cadmium and aluminum tolerance [20-22]. Treatment with GABA abolished aluminum tolerance in wheat roots by inhibiting malate efflux [20]. The report of a GABA-mediated aluminum-activated malate transporter channel in plants provides further proof that GABA is a signaling molecule [16,23]. We recently reported that exogenous GABA treatment alleviated ammonium toxicity in rice seedlings under excessive $\mathrm{NH}_{4}{ }^{+}$conditions by limiting $\mathrm{NH}_{4}{ }^{+}$accumulation and assimilation [24]. However, few investigations on the function of GABA in Fe homeostasis in rice.

In this study, we investigated the effect of GABA on iron homeostasis in rice seedlings. The results will enhance our understanding of the function of GABA in plants and provide useful information on the mediation of Fe homeostasis in rice.

\section{Results}

\subsection{GABA Participates in the Regulation of Iron Homeostasis}

In order to explore the involvement of GABA in the control of Fe homeostasis in rice seedlings, the effects of the GABA $(0.25-1 \mathrm{mM})$ on the chlorophyll concentrations in rice seedlings under conditions of FS and FD were studied. Fe deprivation for two weeks caused apparent chlorosis in newly formed leaves in rice seedlings (Figure 1a). Compared with FS treatment, growth in FD medium reduced the average chlorophyll concentration in the youngest leaves by $33.39 \%$, as determined using a soil-plant analysis development (SPAD) meter (Figure 1b). Application of GABA to seedlings under FD further enhanced leaf chlorosis. Under conditions of FD, 0.5 mM GABA decreased the SPAD value of seedlings from 22.98 to 15.26 (Figure $1 b$ ). GABA treatment also decreased the SPAD value under conditions of FS, and the addition of $0.5 \mathrm{mM} \mathrm{GABA}$ decreased the SPAD value by $16.52 \%$ (Figure $1 b$ ). As $0.5 \mathrm{mM}$ GABA had a moderate effect, this amount was used for further testing.

To investigate if endogenous GABA participates in Fe homeostasis, OsGAD3, was overexpressed. The relative expression levels of OsGAD3 in the two over-expression lines OX16 and OX28 were significantly higher than that in wild type (Nipponbare, NIP) and GABA contents in OX16 and OX28 were respectively 1.24 and 1.59 times of NIP (Figure 2a,b). The two lines were grown in conditions of FS and FD and found that the SPAD values were lower in the youngest leaves of OX16 and OX28 than that of NIP irrespective of Fe supply. Under conditions of FD the SPAD values in OX16 and OX28 were 4.73 and 4.86, respectively, while it was 5.86 in NIP (Figure 2c). 
a

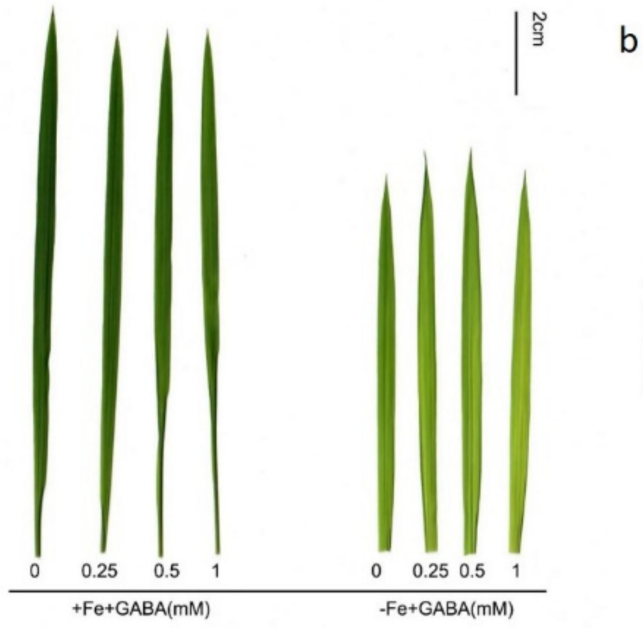

b

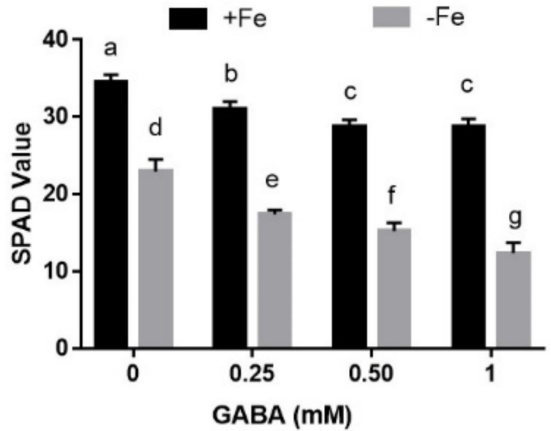

Figure 1. Morphological responses in the fully expanded youngest leaf treated by $\gamma$-Aminobutyric acid (GABA) under conditions of Fe-sufficiency (FS) or -deficiency (FD). Two-week-old seedlings were treated with different concentrations of GABA with or without $\mathrm{FeSO}_{4}(20 \mu \mathrm{M})$ for two weeks. (a) Photos of leaves in rice seedlings grown in different conditions. (b) The soil-plant analysis development (SPAD) value of leaves in rice plants. Data are the means $\pm \operatorname{SD}(n=10)$. Different letters denote a remarkable difference $(p \leq 0.05)$.

a

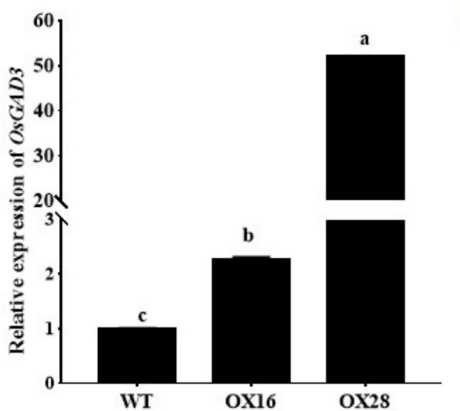

b

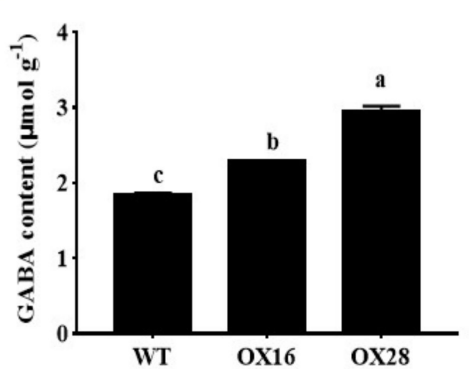

C

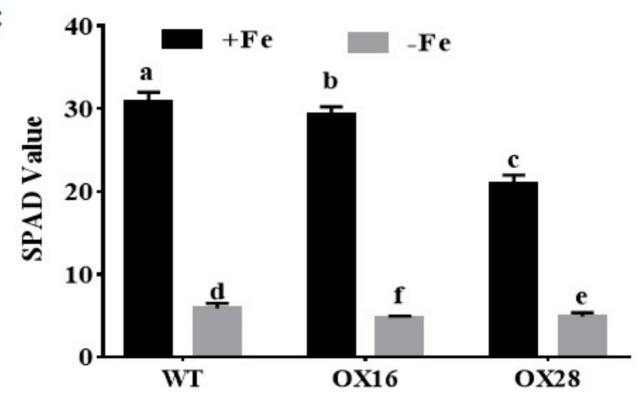

Figure 2. Relative expression of OsGAD3, GABA contents and the SPAD Value in wild type and over-expression lines OX16 and OX28. (a) Relative expression of OsGAD3. (b) GABA contents. Data are the means \pm SD (n=3). (c) The SPAD value in different lines under conditions of FS and FD. Data are the means $\pm \operatorname{SD}(n=10)$. Different letters denote a remarkable difference $(p \leq 0.05)$.

\subsection{GABA Treatment Suppressed Fe Transportation from Roots to Shoots}

To explore how GABA contributes to Fe homeostasis, the Fe content of GABA-treated rice seedlings grown under conditions of FS and FD was measured. Compared with FS treatment, FD decreased Fe concentrations in roots and shoots. The application of GABA reduced the Fe concentrations in the youngest leaves, shoots, and stems, but increased the Fe concentration in roots, under conditions of FS and FD (Figure 3). Under conditions of FD, the addition of $0.5 \mathrm{mM}$ GABA led to a $45.83 \%$ reduce of the Fe accumulation in shoots and a $27.27 \%$ increase in roots compared with the control (Figure 3b,c). Fe level did not differ remarkably between GABA-treated and control whole seedlings, irrespective of Fe supply (Figure 3e). We also found that the Fe content in xylem sap was substantially lesser in GABA-treated than in control seedlings (Figure 3f). 

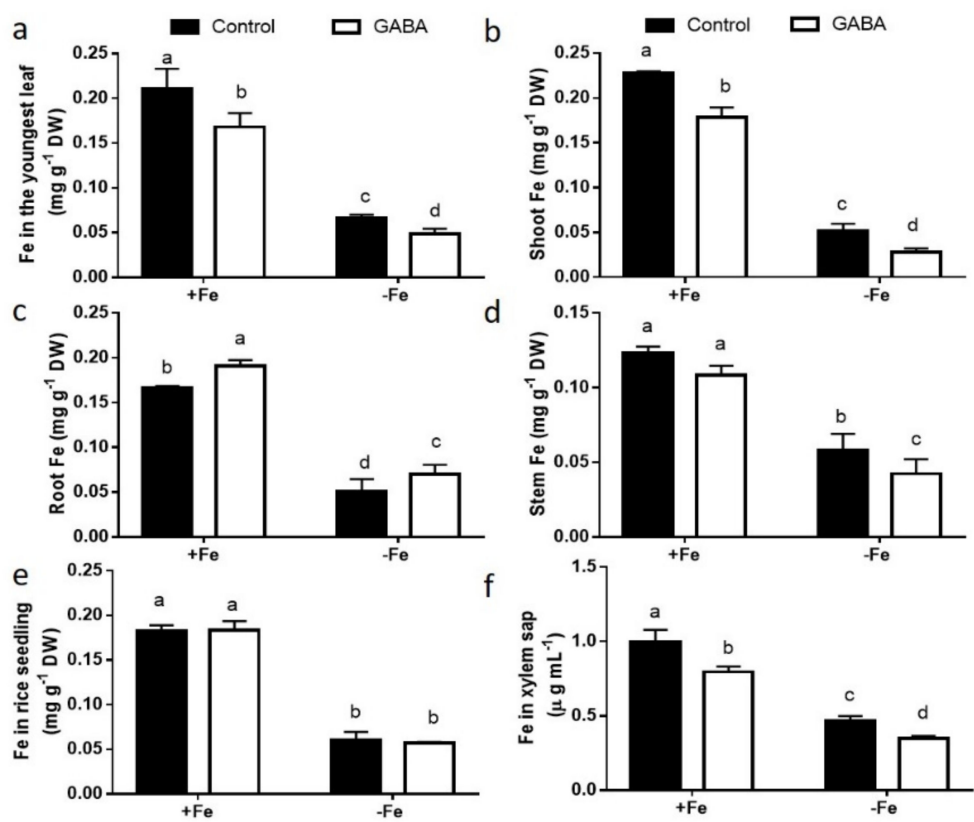

Figure 3. Effect of GABA treatment on Fe concentration. (a) Fe in the youngest leaf. (b) Fe in shoot. (c) Fe in root. (d) Fe in stem. (e) Fe in rice seedling. (f) Fe in xylem sap. The concentration of GABA is $0.5 \mathrm{mM}$. Treatments and statistical analysis were the same as that in Figure 1.

\subsection{GABA Regulated Epidermis-Pericycle Fe Translocation in Roots}

$\mathrm{Fe}^{3+}$ precipitation in roots under conditions of FS with and without GABA treatment was investigated using Perls' blue staining [25,26]. The colour in the epidermal cells of roots is darker than that of treated with GABA. In contrast, $\mathrm{Fe}^{3+}$ was deposited in the pericycle cells of control roots (Figure 4). These staining results demonstrate that GABA regulates Fe translocation from the epidermis to the pericycle.

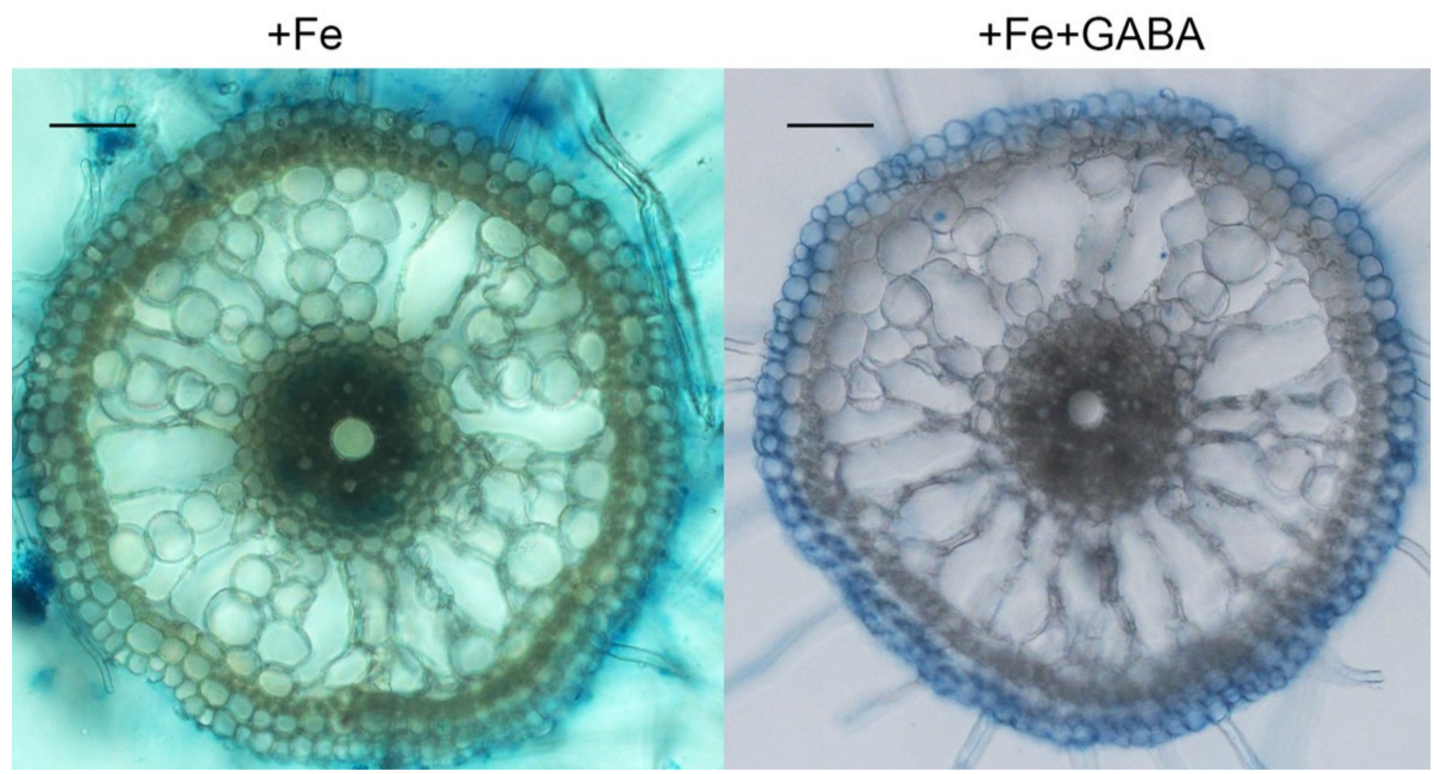

Figure 4. Effect of GABA on ferric accumulation in the roots under conditions of FS. Three-day-old seedlings were treated with $0.5 \mathrm{mM}$ GABA for one week and then roots were stained with Perls' blue stain to visualize ferric accumulation. Hand-cut sections were observed and pictured. Bars $=50 \mu \mathrm{m}$. 


\subsection{GABA Induced Aerenchyma Formation}

To study the effect of GABA on aerenchyma formation, we determined the percentages of aerenchyma area to cortical cell area in each cross section by software-based analysis of microphotos of root cross sections. Compared with the control, GABA increased the percentages of aerenchyma area by $120.95 \%$ under FS conditions (Figure 5). Under conditions of FD, the percentage of aerenchyma in sections of roots treated with GABA was 2.43 times that in controls (Figure. 5).
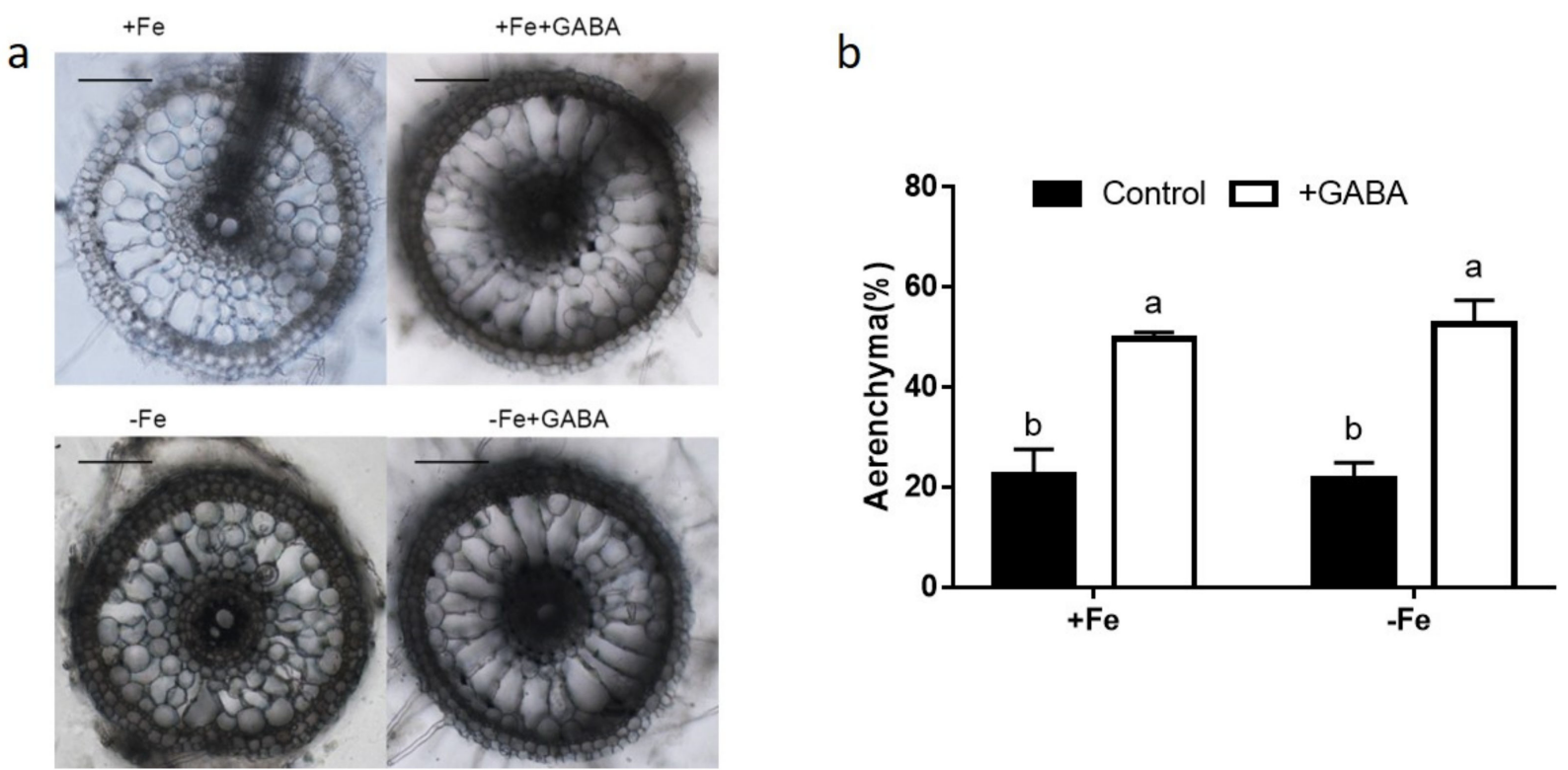

Figure 5. Effect of GABA on the formation of aerenchyma in the roots with or without Fe supply. (a) Root sections of seedlings treated with GABA with or without Fe. (b) GABA induced aerenchyma formation under conditions of both FS and FD. Three-day-old seedlings were treated with $0.5 \mathrm{mM} \mathrm{GABA}$ for one week. Free-hand sections were made $6-8 \mathrm{~cm}$ from root tips. Data are averages $( \pm \mathrm{SD})$ from 5 sections. Bars $=100 \mu \mathrm{m}$.

\subsection{Transcriptomic Analysis of Roots Treated with GABA Under conditions of FD and FS}

To figure out the function of GABA in Fe homeostasis, we investigated the transcriptomes of roots treated with and without $0.5 \mathrm{mM} \mathrm{GABA}$ under conditions of FD and FS for 3 days by RNA sequencing (RNA-seq). In total, $88.36 \mathrm{~Gb}$ clean data for 12 samples was obtained. The percentage of nucleotides with sequencing quality values $\geq Q 30$ in each clean data set was $\geq 92.78 \%$. Samples with $73.79-90.14 \%$ clean reads were mapped successfully onto the rice reference genome; $79.95 \%$ of total reads in roots was uniquely mapped (Table S1), suggesting that the sequences obtained were of high quality and suitable for subsequent bioinformatics analysis.

Differentially expressed genes (DEGs) were analyzed in samples treated with and without GABA under conditions of FS and FD and compared with the reference of samples grown under conditions of FS using DESeq software (adjusted $p \leq 0.05$, fold change $\geq 2$ ). In total, 403 genes were identified as differentially expressed between roots treated with GABA under FS conditions (FSG) and FS. Only 227 DEGs were identified in samples of roots grown under conditions of FDrelative to FS samples. The greatest difference (827 DEGs) was between roots treated with GABA under conditions of FD (FDG) and FS (Figure 6a). 

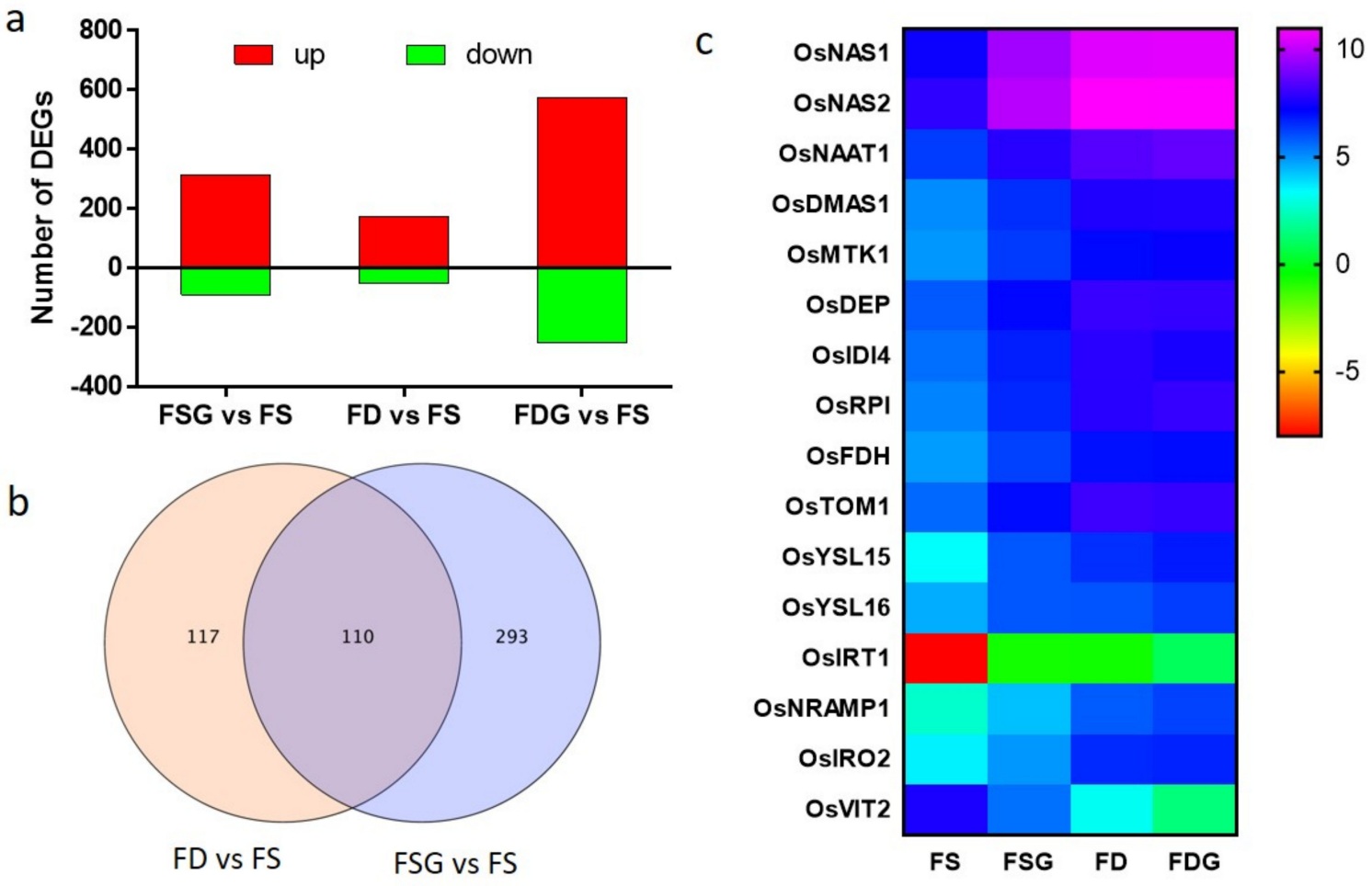

Figure 6. Overview of the changes in transcripts in roots treated with GABA with or without Fe supply. (a) Numbers of differentially expressed genes (DEGs) in roots treated with GABA under conditions of Fe-sufficiency (FSG) or -deficiency (FDG) compared to the control (FS). (b) Venn diagram of genes regulated by Fe deficiency (FD vs. FS) or GABA (FSG vs. FS) in roots. (c) Expression profiles of iron-related genes in roots. The gene -normalized signal intensities are shown in the heat maps using a log2 scale. Two-week-old seedlings were grown in the medium containing $0.5 \mathrm{mM}$ GABA with or without 20 $\mu \mathrm{M} \mathrm{FeSO}_{4}$ for three days.

\subsection{FD-Related Genes Were Induced in GABA-Treated Roots Under Conditions of FS}

The analysis of Gene Ontology (GO) enrichment of the DEGs (FSG vs. FS and FD vs. FS) was performed. Six GO terms were remarkably enriched in DEGs (FD vs. FS): transport (GO:0006810), establishment of localization (GO:0051234), localization (GO:0051179), cellular amino acid and derivative metabolic process (GO:0006519), transport activity (GO:0005215), and membrane (GO:0016020). These GO terms were also enriched in DEGs (FSG vs. FS) (Supplementary Figure S1).

We then analyzed genes regulated by both FD and GABA application. In total, 110 genes in roots were regulated by these two treatments (Figure 6b). Among them, 16 genes are involved in Fe acquisition, transportation, or regulation (Figure 6c). Genes such as OsNAS1, OsNAS2, OsNAAT1, and OsDMAS1 are responsible for MA production and were upregulated in FD and FDG samples (Figure 6c). MAs were produced from SAM and genes involved in methionine cycle were reported to be induced by FD [1,27]. Genes encoding enzymes participating in the methionine cycle, such as methylthioribose kinase 1 (OsMTK1), dehydratase-enolase-phosphatase (OsDEP), aromatic aminotransferase (OsIDI4), ribose 5-phosphate isomerase (OsRPI), and formate dehydrogenase (OsFDH), are also induced by both FD and FSG treatments (Figure 6c). The transporter OsTOM1 is involved in DMA secretion into the rhizosphere [5]. The expression levels of OsTOM1 and genes mediated transporting the Fe (III)-DMA complex, such as OsYSL15 and OsYSL16 [1,2,7], showed upregulation in FD and FSG samples (Figure 6c). The transcript levels of genes mediating $\mathrm{Fe}^{2+}$ transport in the plasma membrane, including OsIRT1 and OsNRAMP1 [11-13], were upregulated in roots treated with GABA or under conditions of FD (Figure 6c). OsIRO2, a positive regulator of various genes related to Fe uptake [28], was upregulated in FD and FSG samples (Figure 6c). OsVIT2, reported to transport Fe across the tonoplast into the 
vacuole [29], was down-regulated in FD and FSG samples (Figure 6c).

To validate the RNA-seq data, four Fe-related genes (OsNAAT1, OsNAS1, OsDMAS1, and OsIRO2) were selected for qRT-PCR measurement of transcripts from roots three days after treatment. All four genes showed the same trends in RNA fold changes measured by both methods (Supplementary Figure S2), supporting the reliability of the RNA-seq data.

\section{Discussion}

Increasing evidence demonstrates the role of GABA as a signaler controlling growth and development and the responses to abiotic stresses in plant $[14,15,17,30]$. However, there are few reports on the function of GABA in the control of nutrient uptake and translocation. Previously, we reported that $3 \mathrm{mM}$ GABA significantly reduced ammonium toxicity in rice seedlings [24]. Sheteiwy et al. [31] reported that $0.5 \mathrm{mM} \mathrm{GABA}$ alleviated oxidative injury caused by salinity in rice seedlings. In the present study, GABA $(0.25-1 \mathrm{mM})$ was used to study the function of GABA in Fe homeostasis in rice seedlings.

FD is known to cause characteristic leaf chlorosis in plants, as Fe is required for the biosynthesis of chlorophyll [1,2]. Exogenous GABA treatment caused leaf chlorosis under conditions of FS and enhanced the symptom of FD-induced leaf chlorosis (Figure 1), suggesting that GABA participated in the control of Fe homeostasis. In contrast to our result, Guo et al. [32] recently reported that foliar application of $20 \mathrm{mM} \mathrm{GABA}$ increased resistance to FD in cucumber. We speculate that this discrepancy is due to difference of species. Rice utilizes the strategy II mechanism to uptake Fe, while cucumber plants uptake Fe by strategy I [1]. Furthermore, overexpression of OsGAD3 gene, encoding a crucial enzyme of GABA production, elevated GABA level and caused lower chlorophyll concentration than wild type under conditions of both FS and FD (Figure 2). These results suggest that GABA is involved in the regulation of Fe homeostasis.

Furthermore, we found that GABA reduced the Fe accumulation in shoots and xylem sap, but elevated the Fe concentration in roots, under conditions of FS and FD (Figure 3). We also found that GABA treatment did not affect Fe content in the whole seedling compared with the control (Figure 3), suggesting that GABA may not increase Fe absorption from soil, but regulates Fe transportation from roots to shoots. In accordance with the increased Fe concentration in roots treated with GABA, we found that more $\mathrm{Fe}^{3+}$ was deposited in the epidermal cells of GABA-treated roots than in control roots, in which $\mathrm{Fe}^{3+}$ accumulated in the pericycle cells using Perls' blue staining (Figure 4). This result suggests that more $\mathrm{Fe}^{2+}$ was oxidized into $\mathrm{Fe}^{3+}$ in the epidermal cells of GABA-treated roots, as $\mathrm{FeSO}_{4}$ was supplied in this study. $\mathrm{Fe}^{2+}$ is more soluble than $\mathrm{Fe}^{3+}$, but presents a risk of oxidative damage in plants when it accumulates at higher concentrations [3]. However, $\mathrm{Fe}^{3+}$ often presents as a constituent of oxides and hydroxide polymers and is not useful for plants due to its low solubility [3]. These results suggest that GABA treatment may cause the oxidation of soluble $\mathrm{Fe}^{2+}$ into insoluble $\mathrm{Fe}^{3+}$ in the epidermal cells of roots and inhibit the transportation of Fe from the epidermis to the pericycle, thereby inhibiting the transportation of Fe from roots to shoots. The mechanism needs to be further studied.

In total, 110 genes were found to be regulated by both GABA and FD. These genes include those related to MA biosynthesis (e.g., OsNAS, OsNAAT, and OsDMAS), the methionine cycle (e.g., OsMTK1, OsDEP, and OsFDH), Fe acquisition and translocation (e.g., OsTOM1, OsYSL15, and OsYSL16), and the regulation of Fe homeostasis (e.g., OsIRO2 and OsVIT2) (Figure 6). These genes are regulated by FD as described previously $[1,2,9,33,34]$. Consistently, GO analysis found that six GO terms were enriched in DEGs of both FSG vs. FS and FD vs FS (Figure S1). These results show that GABA application under FS conditions induced the upregulation of many FD-related genes, suggesting that GABA application under FS conditions causes FD. These findings further support the concept that GABA is a crucial regulator of $\mathrm{Fe}$ homeostasis.

Compared with controls, rice roots treated with GABA showed more aerenchyma formation, irrespective of Fe supply (Figure 5). The aerenchyma allows $\mathrm{O}_{2}$ translocation from shoots to roots and releases $\mathrm{O}_{2}$ to the rhizosphere [35]. The increased oxygen level 
triggered more $\mathrm{Fe}^{2+}$ oxidization to $\mathrm{Fe}^{3+}$ in epidermal cells [36]. Our results show that GABA application induced aerenchyma formation and resulted in $\mathrm{Fe}^{3+}$ production in roots. It also lowered the bioavailability of Fe in roots and subsequently decreased the transportation of Fe from the epidermis to the pericycle. Previous studies reported that ethylene (ETH) and reactive oxygen species (ROS) participated in aerenchyma formation in rice roots [37]. GABA was reported to induce ETH production [38] and GABA can reduce $R O S$ accumulation by increasing activities of antioxidant enzymes under various stresses [31]. However, SIGABA-Ts- silenced tomato plants increased GABA level and enhanced accumulation of ROS under salt stress [39]. Therefore, it will be interesting to measure ETH and ROS levels in roots and to clarify if ETH and ROS participated in GABA-induced aerenchyma formation in the future research.

GABA content increases three to four times within a few hours under anoxic conditions [14,40]. However, Zhang et al. [41] reported that the tolerance of barley to waterlogging is related to more rapid aerenchyma formation, but is not related to increased GABA content in roots. This result is contrary to our finding that GABA induces more aerenchyma formation in rice. This discrepancy may be due to the examination of different species; rice constitutively develops aerenchyma in roots, whereas barley does not develop aerenchyma under well-drained conditions [42].

Excessive amounts of $\mathrm{Fe}^{2+}$ may catalyze the generation of active oxygen species and cause damage to plants $[3,36,43]$. The exclusion of Fe from the roots is a major adaptive mechanism employed by some plants, which develop extensive aerenchyma and oxide excess $\mathrm{Fe}^{2+}$ to $\mathrm{Fe}^{3+}$ in the rhizosphere [3,36]. In this study, we found that GABA can induce aerenchyma formation and inhibit Fe transportation from roots to shoots. Therefore, GABA may be involved in the response of rice to excess $\mathrm{Fe}^{2+}$.

\section{Materials and Methods}

\subsection{Plant Materials and GABA Treatment}

Rice (Oryza sativa L. cv. Huaixianggeng) seeds were imbibed in distilled water for 3 days and then pre-cultured hydroponically with Kimura B solution containing $20 \mu \mathrm{M}$ $\mathrm{FeSO}_{4}$. Seedling (15 days old) were grown in the Kimura B solutions with or without GABA $(0.25,0.5$, and $1 \mathrm{mM})$ under FS or FD conditions. FD was initiated on day 15 after germination by omitting $20 \mu \mathrm{M} \mathrm{FeSO}$ from the culture medium. The seedlings were cultivated in a chamber under 16 -h light and 8 -h dark at $26^{\circ} \mathrm{C}$. The culture medium was changed every 3 days, and the seedlings were harvested after 2 weeks. The experiments were repeated three times with three replicates each.

\subsection{Rice Transformation}

The OsGAD3 (LOC_Os03g13300) coding sequence was amplified and cloned into the pBWA(V)HU, a binary expression vector with a ubiquitin promoter. The fusion vector was transformed into calli of NIP by Agrobacterium-mediated transformation [44]. The overexpression transgenic plants were identified according to the results of PCR using primers (5' - CATACGCTATTTATTTGCTTGG-3' and 5' - CTCGTCGTTGATGATCTGGT-3').

\subsection{Determination of $G A B A$}

Shoot samples were ground using a chilled pestle and mortar in liquid nitrogen, and GABA was extracted according to Kim et al. [45]. GABA was analyzed by an automated amino acid analyzer (Hitachi L-8900, Hitachi High-Technologies Corp., Tokyo, Japan).

\subsection{Analysis of Xylem Sap}

Xylem sap was collected and analyzed two weeks after treatment. The xylem sap was collected as described previously [25]. The concentration of Fe was determined using inductively coupled plasma-mass spectrometry (NexION 300D; PerkinElmer Inc., Shelton, CT, USA). 


\subsection{Analysis of Chlorophyll Content}

SPAD values in the youngest leaves were detected with a portable chlorophyll meter (SPAD-502; Minolta Camera Co., Osaka, Japan).

\subsection{Detedmination of Fe Concentration}

Samples were collected two weeks after treatment and then dried and digested as described previously [46]. Fe was measured by inductively coupled plasma-optical emission spectrometry (Optima 8000; PerkinElmer Inc., Shelton, CT, USA).

\subsection{Perls' Blue Staining}

Seeds were germinated in distilled water for 3 days and then placed into Kimura B solution with or without $0.5 \mathrm{mM}$ GABA under conditions of FS. Perls' blue staining was performed as described previously [25]. One week after treatment, the roots were immersed in the staining solution and vacuum infiltrated for $15 \mathrm{~min}$. The roots were washed with water, and hand-cut sections were then observed and photographed under an optical microscope (Olympus Optical Co. Ltd., Tokyo, Japan).

\subsection{Analysis of Aerenchyma in Roots}

Freehand cross sections were made with a razor blade $6-8 \mathrm{~cm}$ from the primary root tips of seedlings at 7 days after treatment. Sections were pictured with an optical microscope. Aerenchyma formation was estimated by the percentage of cross section possessed by aerenchyma [37]. The areas were measured with Image J (ver. 1.52s).

\subsection{RNA-seq and Analysis}

Total RNA was extracted from the roots 3 days after treatment. This experiment has three biological replicates. The samples were sent to the BMK Company (Beijing, China) for transcriptome sequencing using the Illumine HiSeq2500 platform. Removing low-quality reads, reads containing adapters, and reads containing Poly-N from raw data to obtain clean date. The clean reads were mapped to the rice reference genome IRGSP1.0. Gene expression levels were expressed as fragments per kilobase of transcript per million fragments mapped. DEGs were identified using DESeq. Using the Benjamini and Hochberg approach to adjust $p$ values for control of the false discovery rate (FDR). Genes with FDR $<0.05$ and $\log 2$ (fold change) $\geq 1$ were considered as significantly differential expressed. The analysis of Gene Ontology enrichment of DEGs (FSG vs. FS and FD vs. FS) was conducted by agriGO v2.0 [47].

\subsection{Quantitative Real-Time RT-PCR}

Roots were ground into powder in the presence of liquid nitrogen for total RNA extraction. RT-qPCR was performed as described previously [48]. Actin was used as an internal control to normalize the data. The primers for qRT-PCR in this study were referred to previously report [49].

\subsection{Statistical Analysis}

Statistical analyses were conducted using SPSS (ver. 17.0; SPSS Inc., Chicago, IL, USA). remarkable differences between treatments were identified by $p \leq 0.05$.

\section{Conclusions}

In conclusion, we demonstrate that GABA is involved in the mediation of Fe homeostasis in rice seedlings (Figure 7). GABA treatment induced aerenchyma formation, the oxidization of soluble $\mathrm{Fe}^{2+}$ into insoluble $\mathrm{Fe}^{3+}$, and $\mathrm{Fe}^{3+}$ precipitation in the epidermal cells of roots. GABA inhibited Fe translocation from the epidermis to the pericycle in roots and subsequently decreased the transportation of Fe from roots to shoots, thereby reducing the Fe content in shoots. These findings will be useful for the mediation of Fe homeostasis in rice seedlings. 


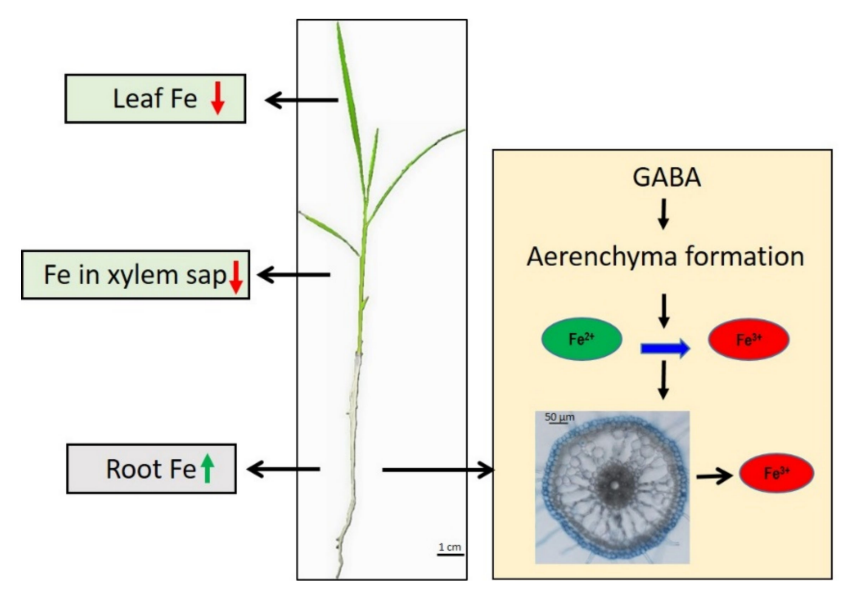

Figure 7. A proposed model for GABA-mediated Fe homeostasis in rice seedlings. GABA treatment induced aerenchyma formation in roots and oxidized soluble $\mathrm{Fe}^{2+}$ into insoluble $\mathrm{Fe}^{3+}$ and $\mathrm{Fe}^{3+}$ was precipitated in epidermal cells of roots, and therefore GABA inhibited the transportation of Fe from roots to shoots. The green arrow denotes increase of Fe content and the red arrows denote decrease of Fe content. The blue arrow denotes $\mathrm{Fe}^{2+}$ was oxidized into $\mathrm{Fe}^{3+}$.

Supplementary Materials: Supplementary materials can be found at https://www.mdpi.com/1422 $-0067 / 22 / 1 / 220 /$ s1.

Author Contributions: Conceptualization, L.G. and C.Z.; methodology, J.W. and H.N.; software, N.Y. and H.N.; validation, L.G. and Q.Q.; formal analysis, Q.Q. and L.G.; investigation, Q.Q., C.Z. and H.N.; resources, C.Z.; data curation, Q.Q. and H.N.; writing-original draft preparation, C.Z. and L.G.; writing-review and editing, L.G.; visualization, Q.Q.; supervision, L.G.; project administration, L.G.; funding acquisition, C.Z. All authors have read and agreed to the published version of the manuscript.

Funding: This research was funded by the National Natural Science Foundation of China (No. 31501236).

Institutional Review Board Statement: Not applicable.

Informed Consent Statement: Not applicable.

Data Availability Statement: The data presented in this study are available in request from the corresponding author.

Acknowledgments: The authors thank Luqing Zheng (Nanjing Agricultural University, Nanjing, China) for useful discussions.

Conflicts of Interest: The authors declare no conflict of interest.

\begin{tabular}{ll}
\multicolumn{2}{l}{ Abbreviations } \\
GABA & $\gamma$-Aminobutyric acid \\
Fe & Iron \\
GAD & Glutamate decarboxylase \\
IRT & Iron-regulated transporters \\
MA & Mugineic acid \\
YSL & Yellow stripe/yellow stripe 1-like \\
NAS & Nicotianamine synthase \\
NAAT & Nicotianamine aminotransferase \\
DMAS & Deoxymugineic acid synthase \\
DMA & Deoxymugineic acid \\
RNA-seq & RNA sequencing \\
DEGs & Differentially expressed genes \\
FS & Fe-sufficiency \\
FSG & with GABA under Fe-sufficient conditions \\
FD & Fe-deficiency \\
FDG & with GABA under Fe-deficient conditions
\end{tabular}




\section{References}

1. Kobayashi, T.; Nishizawa, N.K. Iron uptake, translocation, and regulation in higher plants. Annu. Rev. Plant Biol. 2012, 63, 131-152. [CrossRef] [PubMed]

2. Li, Q.; Chen, L.; Yang, A. The molecular mechanisms underlying iron deficiency responses in rice. Int. J. Mol. Sci. 2020, 21, 43. [CrossRef] [PubMed]

3. Onaga, G.; Drame, K.; Ismail, A. Understanding the regulation of iron nutrition: Can it contribute to improving iron toxicity tolerance in rice? Funct. Plant Biol. 2016, 43, 709-726. [CrossRef] [PubMed]

4. Bashir, K.; Hanada, K.; Shimizu, M.; Seki, M.; Nakanishi, H.; Nishizawa, N. Transcriptomic analysis of rice in response to iron deficiency and excess. Rice 2014, 7, 18. [CrossRef]

5. Nozoye, T.; Nagasaka, S.; Kobayashi, T.; Takahashi, M.; Sato, Y.; Uozumi, N.; Nakanishi, H.; Nishizawa, N. Phytosiderophore efflux transporters are crucial for iron acquisition in graminaceous plants. J. Biol. Chem. 2011, 286, 5446-5454. [CrossRef]

6. Curie, C.; Panaviene, Z.; Loulergue, C.; Dellaporta, S.L.; Briat, J.F.; Walker, E.L. Maize yellow stripe1 encodes a membrane protein directly involved in Fe(III) uptake. Nature 2001, 409, 346-349. [CrossRef]

7. Inoue, H.; Kobayashi, T.; Nozoye, T.; Takahashi, M.; Kakei, Y.; Suzuki, K.; Nakazono, M.; Nakanishi, H.; Mori, S.; Nishizawa, N.K. Rice OsYSL15 is an iron-regulated iron(III)-deoxymugineic acid transporter expressed in the roots and is essential for iron uptake in early growth of the seedlings. J. Biol. Chem. 2009, 284, 3470-3479. [CrossRef]

8. Masuda, H.; Shimochi, E.; Hamada, T.; Senoura, T.; Kobayashi, T.; Aung, M.S.; Ishimaru, Y.; Ogo, Y.; Nakanishi, H.; Nishizawa, N.K. A new transgenic rice line exhibiting enhanced ferric iron reduction and phytosiderophore production confers tolerance to low iron availability in calcareous soil. PLoS ONE 2017, 12, e0173441. [CrossRef]

9. Kobayashi, T.; Nozoye, T.; Nishizawa, N.K. Iron transport and its regulation in plants. Free Radic. Biol. Med. 2019, 133, 11-20. [CrossRef]

10. Ishimaru, Y.; Masuda, H.; Bashir, K.; Inoue, H.; Tsukamoto, T.; Takahashi, M.; Nakanishi, H.; Aoki, N.; Hirose, T.; Ohsugi, R.; et al. Rice metal-nicotianamine transporter, OsYSL2, is required for the long-distance transport of iron and manganese. Plant J. 2010, 62, 379-390. [CrossRef]

11. Bughio, N.; Yamaguchi, H.; Nishizawa, N.; Nakanishi, H.; Mori, S. Cloning an iron-regulated metal transporter from rice. J. Exp. Bot. 2002, 53, 1677-1682. [CrossRef] [PubMed]

12. Ishimaru, Y.; Suzuki, M.; Tsukamoto, T.; Suzuki, K.; Nakazono, M.; Kobayashi, T.; Wada, Y.; Watanabe, S.; Matsuhashi, S.; Takahashi, M.; et al. Rice plants take up iron as an $\mathrm{Fe}^{3+}$-phytosiderophore and as Fe ${ }^{2+}$. Plant J. 2006, 45, 335-346. [CrossRef]

13. Takahashi, R.; Ishimaru, Y.; Senoura, T.; Shimo, H.; Ishikawa, S.; Arao, T.; Nakanishi, H.; Nishizawa, N. The OsNRAMP1 iron transporter is involved in Cd accumulation in rice. J. Exp. Bot. 2011, 62, 4843-4850. [CrossRef] [PubMed]

14. Kinnersley, A.; Turano, F. Gamma aminobutyric acid (GABA) and plant responses to stress. Crit. Rev. Plant Sci. 2000, 19, 479-509. [CrossRef]

15. Bouche, N.; Fromm, H. GABA in plants: Just a metabolite? Trends Plant Sci. 2004, 9, 110-115. [CrossRef] [PubMed]

16. Ramesh, S.A.; Tyerman, S.D.; Gilliham, M.; Xu, B. $\gamma$-Aminobutyric acid (GABA) signalling in plants. Cell Mol. Life Sci. 2017, 74, 1577-1603. [CrossRef]

17. Bown, A.; Shelp, B. Plant GABA: Not just a metabolite. Trends Plant Sci. 2016, 21, 811-813. [CrossRef]

18. Bown, A.W.; Shelp, B.J. Does the GABA shunt regulate cytosolic GABA? Trends Plant Sci. 2020, 25, 422-424. [CrossRef]

19. Hijaz, F.; Killiny, N. The use of deuterium-labeled gamma-aminobutyric (D6-GABA) to study uptake, translocation, and metabolism of exogenous GABA in plants. Plant Methods. 2020, 16, 1. [CrossRef]

20. Sasaki, T.; Yamamoto, Y.; Ezaki, B.; Katsuhara, M.; Ahn, S.J.; Ryan, P.R.; Delhaize, E.; Matsumoto, H. A wheat gene encoding an aluminum-activated malate transporter. Plant J. 2004, 37, 645-653. [CrossRef]

21. Seifikalhor, M.; Aliniaeifard, S.; Bernard, F.; Seif, M.; Latifi, M.; Hassani, B.; Didaran, F.; Bosacchi, M.; Rezadoost, H.; Li, T. $\gamma$-Aminobutyric acid confers cadmium tolerance in maize plants by concerted regulation of polyamine metabolism and antioxidant defense systems. Sci. Rep. 2020, 10, 3356. [CrossRef] [PubMed]

22. Kumar, N.; Gautam, A.; Dubey, A.; Ranjan, R.; Pandey, A.; Kumari, B.; Singh, G.; Mandotra, S.; Chauhan, P.; Srikrishna, S.; et al. GABA mediated reduction of arsenite toxicity in rice seedling through modulation of fatty acids, stress responsive amino acids and polyamines biosynthesis. Ecotoxico. Environ. Saf. 2019, 173, 15-27. [CrossRef] [PubMed]

23. Ramesh, S.; Kamran, M.; Sullivan, W.; Chirkova, L.; Okamoto, M.; Degryse, F.; McLaughlin, M.; Gilliham, M.; Tyerman, S. Aluminum-activated malate transporters can facilitate GABA transport. Plant Cell. 2018, 30, 1147-1164. [CrossRef] [PubMed]

24. Ma, X.; Zhu, C.; Yang, N.; Gan, L.; Xia, K. gamma-Aminobutyric acid addition alleviates ammonium toxicity by limiting ammonium accumulation in rice (Oryza sativa) seedlings. Physiol. Plantarum. 2016, 158, 389-401. [CrossRef]

25. Yokosho, K.; Yamaji, N.; Ueno, D.; Mitani, N.; Ma, J.F. OsFRDL1 is a citrate transporter required for efficient translocation of iron in rice. Plant Physiol. 2009, 149, 297-305. [CrossRef]

26. Green, L.S.; Rogers, E.E. FRD3 controls iron localization in Arabidopsis. Plant Physiol. 2004, 136, 2523-2531. [CrossRef]

27. Ogo, Y.; Kakei, Y.; Itai, R.N.; Kobayashi, T.; Nakanishi, H.; Takahashi, H.; Nakazono, M.; Nishizawa, N.K. Spatial transcriptomes of iron-deficient and cadmium-stressed rice. New Phytol. 2014, 201, 781-794. [CrossRef]

28. Ogo, Y.; Itai, R.N.; Inoue, H.; Kobayashi, T.; Suzuki, M.; Takahashi, M.; Mori, S.; Nishizawa, N. Isolation and characterization of IRO2, a novel iron-regulated bHLH transcription factor in graminaceous plants. J. Exp. Bot. 2006, 57, 2867-2878. [CrossRef] 
29. Zhang, Y.; Xu, Y.; Yi, H.; Gong, J. Vacuolar membrane transporters OsVIT1 and OsVIT2 modulate iron translocation between flag leaves and seeds in rice. Plant J. 2012, 72, 400-410. [CrossRef]

30. Ramesh, S.; Tyerman, S.; Xu, B.; Bose, J.; Kaur, S.; Conn, V.; Domingos, P.; Ullah, S.; Wege, S.; Shabala, S.; et al. GABA signalling modulates plant growth by directly regulating the activity of plant-specific anion transporters. Nat. Commun. 2015, 6, 7879. [CrossRef]

31. Sheteiwy, M.S.; Shao, H.B.; Qi, W.C.; Hamoud, Y.A.; Shaghaleh, H.; Khan, N.U.; Yang, R.P.; Tang, B.P. GABA-alleviated oxidative injury induced by salinity, osmotic Stress and their combination by regulating cellular and molecular signals in Rice. Int. J. Mol. Sci. 2019, 20, 22. [CrossRef] [PubMed]

32. Guo, Z.; Du, N.; Li, Y.; Zheng, S.; Shen, S.; Piao, F. Gamma-aminobutyric acid enhances tolerance to iron deficiency by stimulating auxin signaling in cucumber (Cucumis sativus L.). Ecotoxicol. Environ. Saf. 2020, 192, 110285. [CrossRef] [PubMed]

33. Kobayashi, T.; Itai, R.; Nishizawa, N. Iron deficiency responses in rice roots. Rice 2014, 7, 27. [CrossRef] [PubMed]

34. Kobayashi, T.; Nishizawa, N. Iron sensors and signals in response to iron deficiency. Plant Sci. 2014, 224, 36-43. [CrossRef]

35. Joshi, R.; Kumar, P. Lysigenous aerenchyma formation involves non-apoptotic programmed cell death in rice (Oryza sativa L.) roots. Physiol. Mol. Biol. Plants. 2012, 18,1-9. [CrossRef]

36. Aung, M.; Masuda, H. How does rice defend against excess iron? Physiological and molecular mechanisms. Front. Plant Sci. 2020, 11, 1102. [CrossRef]

37. Yamauchi, T.; Yoshioka, M.; Fukazawa, A.; Mori, H.; Nishizawa, N.K.; Tsutsumi, N.; Yoshioka, H.; Nakazono, M. An NADPH oxidase $\mathrm{RBOH}$ functions in rice roots during lysigenous aerenchyma formation under oxygen-deficient conditions. Plant Cell. 2017, 29, 775-790. [CrossRef]

38. Kathiresan, A.; Tung, P.; Chinnappa, C.C.; Reid, D.M. $\gamma$-Aminobutyric acid stimulates ethylene biosynthesis in sunflower. Plant Physiol. 1997, 115, 129-135. [CrossRef]

39. Bao, H.; Chen, X.; Lv, S.; Jiang, P.; Feng, J.; Fan, P.; Nie, L.; Li, Y. Virus-induced gene silencing reveals control of reactive oxygen species accumulation and salt tolerance in tomato by gamma-aminobutyric acid metabolic pathway. Plant Cell Environ. 2015, emph38, 600-613. [CrossRef]

40. Aurisano, N.; Bertani, A.; Reggiani, R. Anaerobic accumulation of 4-aminobutyrate in rice seedlings-causes and significance. Phytochemistry 1995, 38, 1147-1150. [CrossRef]

41. Zhang, X.; Shabala, S.; Koutoulis, A.; Shabala, L.; Johnson, P.; Hayes, D.; Nichols, D.; Zhou, M. Waterlogging tolerance in barley is associated with faster aerenchyma formation in adventitious roots. Plant Soil. 2015, 394, 355-372. [CrossRef]

42. Rajhi, I.; Yamauchi, T.; Takahashi, H.; Nishiuchi, S.; Shiono, K.; Watanabe, R.; Mliki, A.; Nagamura, Y.; Tsutsumi, N.; Nishizawa, N.K.; et al. Identification of genes expressed in maize root cortical cells during lysigenous aerenchyma formation using laser microdissection and microarray analyses. New Phytol. 2011, 190, 351-368. [CrossRef] [PubMed]

43. Becker, M.; Asch, F. Iron toxicity in rice-conditions and management concepts. J. Plant J. Soil Sci. Plant Nutr. 2005, 168, 558-573. [CrossRef]

44. Nishimura, A.; Aichi, I.; Matsuoka, M. A protocol for Agrobacterium-mediated transformation in rice. Nat. Protoc. 2006, 1, $2796-2802$. [CrossRef] [PubMed]

45. Kim, D.W.; Shibato, J.; Agrawal, G.K.; Fujihara, S.; Iwahashi, H.; Kim, d.H.; Shim, I.S.; Rakwal, R. Gene transcription in the leaves of rice undergoing salt-induced morphological changes (Oryza sativa L.). Mol. Cells. 2007, 24, 45-59.

46. Wang, B.; Li, G.; Zhang, W.H. Brassinosteroids are involved in Fe homeostasis in rice (Oryza sativa L.). J. Exp. Bot. 2015, 66, $2749-2761$. [CrossRef]

47. Tian, T.; Liu, Y.; Yan, H.; You, Q.; Yi, X.; Du, Z.; Xu, W.; Su, Z. AgriGO v2.0: A GO analysis toolkit for the agricultural community, 2017 update. Nucleic Acids Res. 2017, 45, W122-W129. [CrossRef]

48. Li, H.; Wu, H.; Qi, Q.; Li, Z.; Chen, S.; Ding, Q.; Wang, Q.; Yan, Z.; Gai, Y.; Jiang, X.; et al. Gibberellins play a role in regulating tomato fruit ripening. Plant Cell Physiol. 2019, 60, 1619-1629. [CrossRef]

49. Yang, A.; Li, Y.; Xu, Y.; Zhang, W. A receptor-like protein RMC is involved in regulation of iron acquisition in rice. J. Exp. Bot. 2013, 64, 5009-5020. [CrossRef] 